\title{
MASS FORMULAS FOR LOCAL GALOIS REPRESENTATIONS AND QUOTIENT SINGULARITIES I: A COMPARISON OF COUNTING FUNCTIONS
}

\author{
MELANIE MACHETT WOOD AND TAKEHIKO YASUDA
}

\begin{abstract}
We study a relation between the Artin conductor and the weight coming from the motivic integration over wild Deligne-Mumford stacks. As an application, we prove some version of the McKay correspondence, which relates Bhargava's mass formula for extensions of a local field and the Hilbert scheme of points.
\end{abstract}

\section{INTRODUCTION}

The paper was motivated by an observation that two formulas appearing in very different subjects look quite similar. One is Bhargava's mass formula Bha07 for a weighted count of étale algebras (up to isomorphism) of fixed degree over a given local field. The other is an explicit formula for a generating function concerning the Hilbert scheme of points, which is essentially due to Ellingsrud and Strømme ES87. In these two formulas, similar polynomials appear and both have partition numbers as coefficients.

According to Kedlaya Ked07, Bhargava's formula is interpreted as a formula about counting local Galois representations. Let $K$ be a local field with residue field $\mathbb{F}_{q}$ and $G_{K}$ its absolute Galois group. Now the formula becomes of the form,

$$
\frac{1}{n !} \cdot \sum_{\rho \in \operatorname{Hom}_{\text {cont. }}\left(G_{K}, S_{n}\right)} q^{-\mathbf{a}(\rho)}=\sum_{m=0}^{n-1} P(n, n-m) \cdot q^{-m} .
$$

Here $\rho$ runs over continuous homomorphisms $G_{K} \rightarrow S_{n}$ and $\mathbf{a}(\rho)$ is the Artin conductor of the induced representation $G_{K} \rightarrow \mathrm{GL}_{n}(\mathbb{C})$. Then, as in Ked07, Woo08, it is natural to look for formulas when the group $S_{n}$ and the function $-\mathbf{a}$ in the left hand side are replaced with something else.

Let $H$ be the Hilbert scheme of $n$ points of $\mathbb{A}_{\mathbb{F}_{q}}^{2}$ and $Z \subset H$ the locus parametrizing subschemes supported at the origin. The cell decomposition of $Z$ in [ES87, CV08, shows that

$$
\sharp Z\left(\mathbb{F}_{q}\right)=\sum_{m=0}^{n-1} P(n, n-m) \cdot q^{m} .
$$

One finds that the right hand side is the same as that of (1.1) except signs of exponents of $q$.

The aim of this paper is to make clearer the relation between formulas (1.1) and (1.2). The key ingredient is the wild McKay correspondence studied in Yas14, Yas. Let $K$ be a local field with residue field $\kappa=\mathbb{F}_{q}, \mathcal{O}_{K}$ its integer ring and $X:=\mathbb{A}_{\mathcal{O}_{K}}^{n} / \Gamma$ the quotient scheme of $\mathbb{A}_{\mathcal{O}_{K}}^{n}$ by an $\mathcal{O}_{K}$-linear faithful action of a finite group $\Gamma$ 
without pseudo-reflections. Suppose that there exists a crepant resolution $Y \rightarrow X$ and let $Z \subset Y$ be the preimage of the origin of $X_{\kappa}:=X \otimes_{\mathcal{O}_{K}} \kappa$.

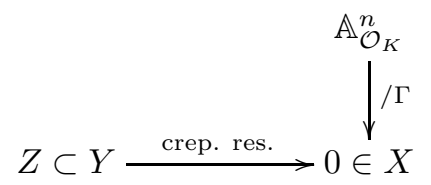

In this setting, we will pose the following variant of what was conjectured in Yas in the motivic context and in the case of algebraically closed residue field:

Conjecture 1.1 (Conjecture 5.17). We have

$$
\sharp Z\left(\mathbb{F}_{q}\right)=\frac{1}{\sharp \Gamma} \cdot \sum_{\rho \in \text { Hom }_{\text {cont. }}\left(G_{K}, \Gamma\right)} q^{\mathbf{w}(\rho)} .
$$

Here $\mathbf{w}(\rho)$ is the weight of $\rho$ (for details, see Section 3 ).

This was proved in Yas14 when $\kappa$ is a finite field of characteristic $p, K=\kappa((t))$, $\Gamma=\mathbb{Z} / p \mathbb{Z}$ and the $\Gamma$-action on $\mathbb{A}_{\kappa[[t]]}^{n}$ is defined over $\kappa$. A particularly interesting situation of the conjecture is when $Y$ is the Hilbert scheme of $n$ points, $H_{\mathcal{O}_{K}}$, now defined over $\mathcal{O}_{K}$ instead of $\mathbb{F}_{q}, X$ is the symmetric product of the affine plane, $S^{n} \mathbb{A}_{\mathcal{O}_{K}}^{2}=\mathbb{A}_{\mathcal{O}_{K}}^{2 n} / S_{n}$ and $\Gamma$ is $S_{n}$. Then the left hand side of the equality in the conjecture is nothing but that of formula (1.2) and the right is the left hand side of (1.1) with $-\mathbf{a}$ replaced with $\mathbf{w}$. Therefore it is natural to ask what the relation between the Artin conductor and the weight is. We will study it in the tame case and also for permutation representations, whether tame or wild. Using an obtained relation and Bhargava's more precise formula [Bha07, we will prove the following:

Theorem 1.2 (Theorem 5.21). The conjecture above holds for $Y=H_{\mathcal{O}_{K}}, X=$ $S^{n} \mathbb{A}_{\mathcal{O}_{K}}^{2}$ and $\Gamma=S_{n}$.

Also we will prove the conjecture when $K$ has equal characteristic prime to $\sharp \Gamma$ and the $\Gamma$-action is defined over the residue field (Corollary 5.19).

From Theorem 1.2 we immediately see that formulas for counts of local Galois representations with respect to $-\mathbf{a}$ and $\mathbf{w}$ interchange by the replacement $q \leftrightarrow q^{-1}$ in this particular case. We will find this duality in a few other cases too. It is still very mysterious why and when the duality holds especially in the wild case. This problem will be discussed in the subsequent paper WY.

The paper is organized as follows. In Sections 2 to 4 , we treat counting functions such as $\mathbf{a}, \mathbf{w}$ and their relatives, and discuss their relations. The last and longest section, Section 5, is devoted to the McKay correspondence. Here we briefly recall the background and formulate several versions of the McKay correspondence both in the tame and wild cases, some of which are still conjectural. We prove some of them in some cases. To formulate and prove a version of the McKay correspondence in the tame case over an arbitrary perfect field, we make heavy use of DeligneMumford stacks. Since this part of the paper is rather independent of the rest, the reader unfamiliar with stacks may skip it.

1.1. Convention. A local field means a complete discrete valuation field with (possibly infinite) perfect residue field, unless otherwise noted. 
1.2. Acknowledgements. Wood was supported by American Institute of Mathematics Five-Year Fellowship and National Science Foundation grants DMS-1147782 and DMS-1301690. Yasuda was supported by Grants-in-Aid for Scientific Research (22740020).

\section{Total masses and the Artin conductor}

2.1. Total masses and counting functions. Let $K$ be a local field and $G_{K}:=$ $\operatorname{Gal}\left(K^{\text {sep }} / K\right)$ its absolute Galois group. For a finite group $\Gamma$, we put $S_{K, \Gamma}$ to be the set of continuous homomorphisms $G_{K} \rightarrow \Gamma$. Given a function $c: S_{K, \Gamma} \rightarrow \mathbb{R}$, the total mass of $(K, \Gamma, c)$ is

$$
M(K, \Gamma, c):=\frac{1}{\sharp \Gamma} \sum_{\rho \in S_{K, \Gamma}} q^{-c(\rho)} \in \mathbb{R}_{\geq 0} \cup\{\infty\},
$$

which was defined by Kedlaya Ked07 for a special choice of $c$ and Wood Woo08] in general. This quantity is the main concern of this paper. We call $c$ a counting function.

We are mainly interested in two choices of the counting function $c$. One is the Artin conductor as studied in Ked07 and the other the weight originating in the study of motivic integration over wild Deligne-Mumford stacks Y Yas, 1 Both are associated to a representation of $\Gamma$. They and their relatives share many properties. For the unified treatment, we will introduce the following notion.

Definition 2.1. For each local field $K$, let us denote by $R_{K}$ either a fixed field (independent of $K$ ), $K$ itself or its integer ring $\mathcal{O}_{K}$. A counting system is a family of functions,

$$
c_{\bullet}=\left(c_{K, \Gamma, \tau}: S_{K, \Gamma} \rightarrow \mathbb{R}\right)_{K, \Gamma, \tau},
$$

where $K$ runs over local fields, $\Gamma$ finite groups and $\tau$ finite-dimensional $\Gamma$-representations $\Gamma \rightarrow \mathrm{GL}_{n}\left(R_{K}\right)$.

Next we introduce several properties of a counting function system.

Definition 2.2. We say that $c_{\bullet}$ is additive if we always have

$$
c_{K, \Gamma, \tau \oplus \sigma}=c_{K, \Gamma, \tau}+c_{K, \Gamma, \sigma}
$$

and if for the trivial representation $\alpha: \Gamma \rightarrow \mathrm{GL}_{1}\left(R_{K}\right)$, we have

$$
c_{K, \Gamma, \alpha} \equiv 0 .
$$

We say that $c_{\bullet}$ is geometric if we always have

$$
c_{K, \Gamma, \tau}(\rho)=c_{K^{\mathrm{nr}}, \Gamma, \tau^{\mathrm{nr}}}\left(\rho^{\mathrm{nr}}\right),
$$

where the superscript "nr" means passing from $K$ to the completion of the maximal unramified extension of $K$, which we denote by $K^{\text {nr }}$ by a slight abuse of notation.

We say that $c_{\bullet}$ is convertible if for a homomorphism $\phi: \Gamma_{1} \rightarrow \Gamma_{2}$ of finite groups and a $\Gamma_{2}$-representation $\tau: \Gamma_{2} \rightarrow \mathrm{GL}_{n}\left(R_{K}\right)$, we always have

$$
c_{K, \Gamma_{1}, \tau \circ \phi}(\rho)=c_{K, \Gamma_{2}, \tau}(\phi \circ \rho) \quad\left(\rho \in S_{K, \Gamma_{1}}\right) .
$$

Finally we say that $c_{\bullet}$ is complete if it is additive, geometric and convertible.

\footnotetext{
${ }^{1}$ When $\Gamma$ is constructed from symmetric groups by using wreath products and direct products, there is yet another interesting choice of the counting function Woo08.
} 
2.2. The Artin conductor. Let $L / K$ be a finite Galois extension of local fields with $G:=\operatorname{Gal}(L / K)$ and $G_{i}, i=0,1, \ldots$, the $i$ th ramification subgroups, which are defined by

$$
G_{i}=\left\{g \in G \mid v_{L}(g x-g) \geq i+1 \text { for all } x \in \mathcal{O}_{L}\right\} .
$$

Here $v_{L}$ is the normalized valuation of $L$. For a representation $\tau: G \rightarrow \mathrm{GL}_{n}(k)$ with $k$ either a fixed field or $K$, following [Ser87, we define the Artin conductor of $\tau$ by

$$
\mathbf{a}_{\tau}(L / K)=\mathbf{a}_{\tau}(L):=\sum_{i=0}^{\infty} \frac{1}{\left(G_{0}: G_{i}\right)} \cdot \operatorname{codim}\left(k^{n}\right)^{G_{i}} .
$$

We write the conductor as a function in $L$ for the consistency with the later use.

Remark 2.3. If $k=\mathbb{C}$, then the definition above coincides with the one using the inner product of characters as defined in Ser79, VI, §2]. Taguchi Tag02 proved that our Artin conductor satisfies the same induction formula as the ordinary one over $\mathbb{C}$ does. In particular, the well-known relation between the Artin conductor and the discriminant still holds in our setting.

We also define the Swan conductor and the tame part (of the Artin conductor) as follows:

$$
\begin{aligned}
\mathbf{s}_{\tau}(L) & :=\sum_{i=1}^{\infty} \frac{1}{\left(G_{0}: G_{i}\right)} \cdot \operatorname{codim}\left(k^{n}\right)^{G_{i}} \text { and } \\
\mathbf{t}_{\tau}(L): & :=\mathbf{a}_{\tau}(L)-\mathbf{s}_{\tau}(L)=\operatorname{codim}\left(k^{n}\right)^{G_{0}} .
\end{aligned}
$$

2.3. The Artin conductor as a counting system. Let $K$ be a local field and fix a representation $\tau: \Gamma \rightarrow \mathrm{GL}_{n}(k)$ with $k=K$ or a field independent of $K$. Then for $\rho \in S_{K, \Gamma}$, we put $L_{\rho} / K$ to be the Galois extension corresponding to the kernel of $\tau \circ \rho$ and $\tau^{\prime}: \Gamma^{\prime} \hookrightarrow \mathrm{GL}_{n}(k)$ to be the induced representation of $\Gamma^{\prime}:=\operatorname{Im}(\tau \circ \rho)$. We define the function

$$
\begin{aligned}
\mathbf{a}_{K, \Gamma, \tau}=\mathbf{a}_{\tau}: S_{K, \Gamma} & \rightarrow \mathbb{Z}_{\geq 0} \\
\rho & \mapsto \mathbf{a}_{\tau}(\rho):=\mathbf{a}_{\tau^{\prime}}\left(L_{\rho}\right),
\end{aligned}
$$

which we again call the Artin conductor. Similarly we define the Swan conductor, $\mathbf{s}_{K, \Gamma, \tau}=\mathbf{s}_{\tau}$, and the tame part of the Artin conductor, $\mathbf{t}_{K, \Gamma, \tau}=\mathbf{t}_{\tau}$, as functions on $S_{K, \Gamma}$. We thus have obtained three counting systems $\mathbf{a}_{\bullet}, \mathbf{s}_{\bullet}$ and $\mathbf{t}_{\bullet}$.

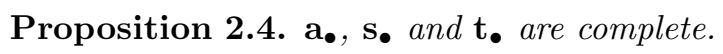

Proof. The additivity and convertibility are obvious from the definition. The geometricity follows from the fact that the higher ramification groups $G_{i}$ of a Galois extension $L / K$ is stable by passing to $L^{\mathrm{nr}} / K^{\mathrm{nr}}$.

The completeness is shared by all counting systems appearing in this paper including the weight introduced below. Let us now see a property of the Artin conductor which distinguish it from the weight.

Lemma 2.5. Let $\alpha: \Gamma \rightarrow \Gamma$ be an automorphism preserving all subgroups and define a map

$$
\alpha^{*}: S_{K, \Gamma} \rightarrow S_{K, \Gamma}, \rho \mapsto \alpha \circ \rho .
$$

Then, for $c=\mathbf{a}, \mathbf{s}$ and $\mathbf{t}$ and for any $\Gamma$-representation $\tau$, we have

$$
c_{\tau}=c_{\tau} \circ \alpha^{*} .
$$


Proof. With the notation as above, the Galois extensions $L_{\rho}$ and $L_{\alpha \circ \rho}$ are identical, which shows the lemma.

2.4. Bhargava's mass formula. Let $K$ be a local field with a finite residue field $\mathbb{F}_{q}$. Serre [Ser78] proved the following mass formula: for a positive integer $n$,

$$
\sum_{L} \frac{1}{\sharp \operatorname{Aut}(L / K)} \cdot q^{-v_{K}\left(d_{L / K}\right)}=q^{1-n},
$$

where $L / K$ runs over the isomorphism classes of totally ramified field extensions with $[L: K]=n$ and $d_{L / K}$ denotes their discriminants. Then Bhargava Bha07. proved an analogous formula for étale extensions: if $P(n, r)$ denotes the number of partitions of $n$ into exactly $r$ parts, then

$$
\sum_{E} \frac{1}{\sharp \operatorname{Aut}(E / K)} \cdot q^{-v_{K}\left(d_{E / K}\right)}=\sum_{m=0}^{n-1} P(n, n-m) \cdot q^{-m},
$$

where $E / K$ runs over the isomorphism classes of étale extensions with $[E: K]=n$.

Let $S_{n}$ be the $n$th symmetric group. Each element $\rho \in S_{K, S_{n}}$ defines a $G_{K}$-action on $\{1,2, \ldots, n\}$ and an étale extension $E_{\rho} / K$ of degree $n$. This gives a one-to-one correspondence between the classes of $\rho \in S_{K, S_{n}}$ modulo the conjugation of $S_{n}$ and the isomorphism classes of étale extensions $E / K$ with $[E: K]=n$. Let

$$
\sigma: S_{n} \rightarrow \mathrm{GL}_{n}(k)
$$

be the defining representation of $S_{n}$ with $k$ an arbitrary field. Kedlaya Ked07. showed that for $\rho \in S_{K, S_{n}}$,

$$
\mathbf{a}_{\sigma}(\rho)=v_{K}\left(d_{E_{\rho} / K}\right)
$$

and that through the correspondence above, Bhargava's formula is rewritten as follows:

$$
M\left(K, S_{n}, \mathbf{a}_{\sigma}\right)=\sum_{i=0}^{n-1} P(n, n-m) \cdot q^{-m} .
$$

We can slightly generalize Equality (2.1) as follows:

Lemma 2.6. Let $\tau: \Gamma \rightarrow \mathrm{GL}_{n}(k)$ be a permutation representation of a finite group $\Gamma$. For $\rho \in S_{K, \Gamma}$, we denote by $E_{\rho} / K$ the corresponding étale extension of degree n. Then

$$
\mathbf{a}_{\tau}(\rho)=v_{K}\left(d_{E_{\rho} / K}\right) .
$$

Proof. From the convertibility of $\mathbf{a}_{\bullet}$, we may replace $\Gamma$ with $S_{n}$ and $\tau$ with its defining representation $\sigma$ without changing either side of the equality. Thus we can reduce to (2.1).

\footnotetext{
${ }^{2}$ In fact, he considers only the case $k=\mathbb{C}$. However, since $\sigma$ is a permutation representation, $\mathbf{a}_{\sigma}$ is independent of $k$. Also, for any representation, Taguchi Tag02 proved that the relation between the Artin conductor and the discriminant is valid over any field.
} 


\section{Weights}

Another function on $S_{K, \Gamma}$, the weight, came from the study of the wild McKay correspondence using motivic integration Yas, (see Section 5).

Let $\Gamma$ be a finite group. As $G_{K}$ is the étale fundamental group of Spec $K$, there is a one-to-one correspondence between $S_{K, \Gamma}$ and the set of pointed étale $\Gamma$-torsors over Spec $K$ modulo isomorphism (see [Mil80, page 44]). As the data of pointing are not so important in what follows, we will often ignore them. We denote the $\Gamma$-torsor corresponding to $\rho \in S_{K, \Gamma}$ as

$$
T_{\rho}=\operatorname{Spec} M_{\rho} \rightarrow \operatorname{Spec} K
$$

Let $\mathcal{O}_{M_{\rho}}$ be the integral closure of $\mathcal{O}_{K}$ in $M_{\rho}$ and $\tau: \Gamma \rightarrow \operatorname{GL}_{n}\left(\mathcal{O}_{K}\right)$ a $\Gamma$ representation. Then $\Gamma$ acts on $\mathcal{O}_{M_{\rho}}^{\oplus n}$ in two ways. First $\Gamma$ acts on $\mathcal{O}_{M_{\rho}}$ and hence diagonally on $\mathcal{O}_{M_{\rho}}^{\oplus n}$, which determines

$$
\delta: \Gamma \rightarrow \operatorname{Aut}_{\mathcal{O}_{K}}\left(\mathcal{O}_{M_{\rho}}\right)^{\oplus n} .
$$

On the other hand, $\tau$ naturally extends to

$$
\tilde{\tau}: \Gamma \rightarrow \mathrm{GL}_{n}\left(\mathcal{O}_{K}\right) \hookrightarrow \mathrm{GL}_{n}\left(\mathcal{O}_{M_{\rho}}\right) .
$$

Definition 3.1. We define the tuning submodule $\Xi_{\rho} \subset \mathcal{O}_{M_{\rho}}^{\oplus n}$ to be the subset of elements on which the two actions $\delta$ and $\tilde{\tau}$ coincide, that is,

$$
\Xi_{\rho}:=\left\{x \in \mathcal{O}_{M_{\rho}}^{\oplus n} \mid \forall \gamma \in \Gamma, \delta(\gamma)(x)=\tilde{\tau}(\gamma)(x)\right\} .
$$

When we want to specify $\tau$, we write it as $\Xi_{\rho}^{\tau}$.

Lemma 3.2 (प्(

Proof. This is obviously a torsion-free, hence free $\mathcal{O}_{K}$-module. To show that this has rank $n$, we take a trivial $\Gamma$-torsor $\operatorname{Spec} N \rightarrow \operatorname{Spec} L$ obtained from (3.1) by a scalar extension $L / K$. Then we write $N=\prod_{\gamma \in \Gamma} L_{\gamma}$ such that $L_{\gamma}$ are copies of $L$ and each $\gamma \in \Gamma$ induces an isomorphism $L_{1} \stackrel{\sim}{\rightarrow} L_{\gamma}$ which is actually the identity of $L$. Then $\Xi_{\rho} \otimes_{\mathcal{O}_{K}} L \subset N^{\oplus n}$ is still the subset of those elements on which the two induced $\Gamma$-actions coincide. Hence

$$
\Xi_{\rho} \otimes_{\mathcal{O}_{K}} L=\left\{(\tau(\gamma)(x))_{\gamma \in \Gamma} \mid x \in L^{\oplus n}\right\} \cong L^{\oplus n} .
$$

This shows that $\Xi_{\rho}$ has rank $n$.

Definition 3.3. We define a counting system $\mathbf{v} \bullet$ as follows: Let $x_{i}=\left(x_{i j}\right)_{1 \leq j \leq n} \in$ $\mathcal{O}_{M_{\rho}}^{\oplus n}, 1 \leq i \leq n$, be a basis of $\Xi_{\rho}$ as a free $\mathcal{O}_{K}$-module. Then we define $\mathbf{v}_{K, \Gamma, \tau}(\rho)=$ $\mathbf{v}_{\tau}(\rho) \in \frac{1}{\sharp \Gamma} \mathbb{Z}_{\geq 0}$ by

$$
\mathbf{v}_{\tau}(\rho):=\frac{1}{\sharp \Gamma} \cdot \text { length }\left(\frac{\mathcal{O}_{M_{\rho}}}{\left(\operatorname{det}\left(x_{i j}\right)\right)}\right)=\frac{1}{\sharp \Gamma} \cdot \operatorname{length}\left(\frac{\mathcal{O}_{M_{\rho}}^{\oplus n}}{\mathcal{O}_{M_{\rho}} \cdot \Xi_{\rho}}\right) .
$$

Lemma 3.4. $\mathbf{v}_{\bullet}$ is complete.

Proof. The additivity is obvious. To show the geometricity, it suffices to show the identity of submodules of $\mathcal{O}_{M_{\rho \mathrm{nr}}}$,

$$
\Xi_{\rho^{\mathrm{nr}}}=\Xi_{\rho} \otimes_{\mathcal{O}_{M_{\rho}}} \mathcal{O}_{M_{\rho^{\mathrm{nr}}}}
$$

Indeed $\Xi_{\rho}$ is defined as the kernel of an $\mathcal{O}_{K}$-linear map

$$
(\delta(\gamma)-\tilde{\tau}(\gamma))_{\gamma \in \Gamma}: \mathcal{O}_{M_{\rho}}^{\oplus n} \rightarrow\left(\mathcal{O}_{M_{\rho}}^{\oplus n}\right)^{\oplus \sharp \Gamma}
$$


and similarly for $\Xi_{\rho^{\mathrm{nr}}}$ : denote them $\alpha$ and $\alpha_{\mathrm{nr}}$. From the compatibilities of tensor product with completion and direct limit, we have that $\mathcal{O}_{M_{\rho}^{\mathrm{nr}}}=\mathcal{O}_{M_{\rho}} \otimes_{\mathcal{O}_{K}} \mathcal{O}_{K^{\mathrm{nr}}}$. We see that $\alpha_{\mathrm{nr}}$ is obtained from $\alpha$ by tensoring $\mathcal{O}_{K^{\mathrm{nr}}}$ over $\mathcal{O}_{K}$, for instance, by looking at the matrix representations of these maps. Therefore $\alpha_{\mathrm{nr}}$ is obtained from $\alpha$ by tensoring $\mathcal{O}_{M_{\rho}^{\mathrm{nr}}}$ over $\mathcal{O}_{M_{\rho}}$. The desired identity holds since $\mathcal{O}_{M_{\rho^{\mathrm{nr}}}}$ is flat over $\mathcal{O}_{M_{\rho}}$.

To show the convertibility, it suffices to show it in the cases where $\phi: \Gamma_{1} \rightarrow \Gamma_{2}$ in Definition 2.2 is respectively injective and surjective. First we consider the former case, supposing that $\Gamma_{1}$ is a subgroup of $\Gamma_{2}$. Let $\rho_{1} \in S_{K, \Gamma_{1}}, \rho_{2}:=\phi \circ \rho_{1} \in S_{K, \Gamma_{2}}$ and

$$
T_{i}=\operatorname{Spec} M_{i} \rightarrow \operatorname{Spec} K \quad(i=1,2)
$$

the corresponding $\Gamma_{i}$-torsors. Then $T_{2}$ is isomorphic to the disjoint union of copies of $T_{1}$. Therefore if $\gamma_{1}=1, \gamma_{2}, \ldots, \gamma_{l} \in \Gamma_{2}$ are representatives of $\Gamma_{1}$-cosets, we can write

$$
\mathcal{O}_{M_{2}}=\prod_{i} \mathcal{O}_{M_{1}, i}
$$

with $\mathcal{O}_{M_{1}, i}$ copies of $\mathcal{O}_{M_{1}}$. Moreover we suppose that for each $i$, the $\gamma_{i}$-action on $\mathcal{O}_{M_{2}}$ induces the isomorphism $\mathcal{O}_{M_{1}, 1} \rightarrow \mathcal{O}_{M_{1}, i}$ coming from the identity of $\mathcal{O}_{M_{1}}$. If $\Xi_{i} \subset \mathcal{O}_{M_{i}}^{\oplus n}, i=1,2$, are the tuning modules, then

$$
\begin{aligned}
\Xi_{2} & =\left\{x \in \mathcal{O}_{M_{2}}^{\oplus n} \mid \forall \gamma \in \Gamma_{2}, \delta(\gamma)(x)=\tau(\gamma)(x)\right\} \\
& =\left\{\left(x_{i}\right) \in \prod_{i} \mathcal{O}_{M_{1}, i}^{\oplus n} \mid x_{1} \in \Xi_{1} \text { and } x_{i}=\gamma_{i}\left(x_{1}\right)\right\} .
\end{aligned}
$$

This shows the convertibility in this case.

Next consider the case where $\phi$ is surjective. We may suppose that $\Gamma_{2}$ is the quotient of $\Gamma_{1}$ by a normal subgroup $N$. With the same notation as above, we have $T_{2}=T_{1} / N$ and $\mathcal{O}_{M_{2}}=\mathcal{O}_{M_{1}}^{N}$. Then we see that $\Xi_{1}$ is contained in $\mathcal{O}_{M_{2}}^{\oplus n}$ and identical to $\Xi_{2}$. This finishes the proof.

Let $\Gamma^{\prime} \subset \Gamma$ be the stabilizer of a connected component of $T_{\rho^{\mathrm{nr}}}=\operatorname{Spec} M_{\rho^{\mathrm{nr}}}$, that is, if $U$ is a connected component, then $\Gamma^{\prime}=\{\gamma \in \Gamma \mid \gamma(U)=U\}$. This subgroup is unique up to conjugacy. Denoting the residue field of $K$ by $\kappa$, we consider the following $\Gamma^{\prime}$-representation over $\kappa$,

$$
\Gamma^{\prime} \hookrightarrow \Gamma \stackrel{\tau}{\rightarrow} \mathrm{GL}_{n}\left(\mathcal{O}_{K}\right) \rightarrow \mathrm{GL}_{n}(\kappa),
$$

and write its fixed point locus as $\left(\kappa^{n}\right)^{\Gamma^{\prime}}$.

Definition 3.5. We define the residual tame part by

$$
\overline{\mathbf{t}}_{\tau}(\rho):=\operatorname{codim}\left(\kappa^{n}\right)^{\Gamma^{\prime}} .
$$

The following is obvious from the definition.

Lemma 3.6. The counting system $\overline{\mathbf{t}}_{\bullet}$ is complete.

Definition 3.7. We define the weight of $\rho \in S_{K, \Gamma}$ with respect to $\tau$ as

$$
\mathbf{w}_{\tau}(\rho):=\overline{\mathbf{t}}_{\tau}(\rho)-\mathbf{v}_{\tau}(\rho) \in \frac{1}{\sharp \Gamma} \cdot \mathbb{Z}_{\leq n} .
$$

Corollary 3.8. The counting system $\mathbf{w}_{\bullet}$ is complete.

Proof. This follows from the completeness of $\mathbf{v}_{\bullet}$ and $\overline{\mathbf{t}}_{\mathbf{\bullet}}$. 


\section{A comparison of the Artin Conductor And the Weight}

For a representation $\tau: \Gamma \rightarrow \mathrm{GL}_{n}\left(\mathcal{O}_{K}\right)$, we define a function $\mathbf{a}_{\tau}$ on $S_{K, \Gamma}$ by $\mathbf{a}_{\tau}:=\mathbf{a}_{\iota \circ \tau}$ with $\iota: \mathrm{GL}_{n}\left(\mathcal{O}_{K}\right) \hookrightarrow \mathrm{GL}_{n}(K)$. We will study a relation among the Artin conductor $\mathbf{a}_{\tau}$, the weight $\mathbf{w}_{\tau}$ and their relatives.

4.1. The tame case. In this subsection, we consider the tame case, that is, $\sharp \Gamma$ is prime to the residue characteristic of $K$. Since the involved counting systems are complete, in particular, geometric, without loss of generality, we now suppose that $K$ has an algebraically closed residue field.

For $\rho \in S_{K, \Gamma}$, let $L_{\rho} / K$ be the Galois extension defined by the kernel of $\tau \circ \rho$. Its Galois group $G:=\operatorname{Gal}\left(L_{\rho} / K\right)$ is cyclic, say having a generator $g$. As for higher ramification groups, we have $G_{0}=G$ and $G_{i}=1$ for $i \geq 1$. Therefore we easily see the following.

Lemma 4.1. We have

$$
\mathbf{a}_{\tau}(\rho)=\mathbf{t}_{\tau}(\rho)=\operatorname{codim}\left(K^{n}\right)^{G} \text { and } \mathbf{s}_{\tau}(\rho)=0 .
$$

Corollary 4.2. If $\tau^{\vee}$ is the dual representation of $\tau$, then

$$
\mathbf{a}_{\tau}=\mathbf{a}_{\tau^{\vee}}
$$

Proof. From the preceding lemma, $\mathbf{a}_{\tau}(\rho)$ is equal to the number of eigenvalues of the $g$-action on $K^{n}$ not equal to 1 . This number does not change by passing to the dual representation, hence the corollary holds.

For a positive integer $l$ and a uniformizer $\pi$ of $K$, we can write $L_{\rho}=K(\sqrt[l]{\pi})$.

Lemma 4.3 (cf. [Yas, Example 6.7]). Let $\zeta \in K$ be the primitive l-th root of unity such that $g(\sqrt[l]{\pi})=\zeta \cdot \sqrt[l]{\pi}$, and let $\zeta^{a_{1}}, \ldots, \zeta^{a_{n}}, 0 \leq a_{i}<l$, be the eigenvalues of $g \in \mathrm{GL}_{n}(K)$. Then

$$
\mathbf{v}_{\tau}(\rho)=\frac{1}{l} \cdot \sum_{i=0}^{n} a_{i}
$$

Proof. We first claim that $g$ is diagonalizable over $\mathcal{O}_{K}$. Let $\xi$ be a power of $\zeta$ and $M_{\xi} \subset\left(\mathcal{O}_{K}\right)^{\oplus n}$ be the $\xi$-eigenmodule of $g$. The inclusion map $M_{\xi} \hookrightarrow\left(\mathcal{O}_{K}\right)^{\oplus n}$ admits a splitting,

$$
\sigma_{\xi}:\left(\mathcal{O}_{K}\right)^{\oplus n} \rightarrow M_{\xi}, x \mapsto \frac{1}{l} \sum_{i=0}^{l-1} g^{i} \xi^{-i} x .
$$

The product of $\sigma_{\zeta^{i}}, 0 \leq i<l$ gives the inverse of

$$
\bigoplus_{i=0}^{l-1} M_{\zeta^{i}} \rightarrow\left(\mathcal{O}_{K}\right)^{\oplus n},\left(x_{i}\right) \mapsto \sum x_{i} .
$$

This shows the claim. If we suppose $g=\operatorname{diag}\left(\zeta^{a_{1}}, \ldots, \zeta^{a_{n}}\right)$, we can choose

$$
\left(\sqrt[l]{\pi}^{a_{1}}, 0, \ldots, 0\right), \ldots,\left(0, \ldots, 0, \sqrt[l]{\pi}^{a_{n}}\right)
$$

as a basis of the tuning submodule $\Xi_{\rho} \subset\left(\mathcal{O}_{L_{\rho}}\right)^{\oplus n}$. This proves the lemma.

Note that if we write the eigenvalues $\zeta^{a_{i}}, 0 \leq a_{i}<l$ as $\zeta^{b_{i}}, 0<b_{i} \leq l$, then

$$
\mathbf{w}_{\tau}(\rho)=\sharp\left\{i \mid a_{i} \neq 0\right\}-\frac{1}{l} \sum_{i=1}^{n} a_{i}=n-\frac{1}{l} \sum_{i=1}^{n} b_{i} .
$$


Definition 4.4. We say that the representation $\tau$ is balanced if for every $\gamma \in \Gamma$, every non-real eigenvalue of $\tau(\gamma)$ appears in a pair with its inverse, that is, $\tau(\gamma)$ have eigenvalues

$$
\epsilon_{1}, \epsilon_{1}^{-1}, \epsilon_{2}, \epsilon_{2}^{-1}, \ldots, \epsilon_{m}, \epsilon_{m}^{-1}, 1, \ldots, 1,-1, \ldots,-1
$$

up to permutation.

The following are examples of balanced representations:

(1) a representation defined over a field which can be embedded in $\mathbb{R}$.

(2) a permutation representation.

(3) a self-dual representation, that is, a representation which is isomorphic to its dual.

(4) $\tau \oplus \tau^{\vee}$ for any $\tau$.

Proposition 4.5. Suppose that $\tau$ is balanced. Then

$$
\mathbf{w}_{\tau}=\mathbf{v}_{\tau}=\frac{1}{2} \cdot \overline{\mathbf{t}}_{\tau}=\frac{1}{2} \cdot \mathbf{t}_{\tau}=\frac{1}{2} \cdot \mathbf{a}_{\tau} .
$$

Proof. With the notation as above, the generator $g$ of $G=\operatorname{Gal}\left(L_{\rho} / K\right)$ has eigenvalues

$$
\zeta^{b_{1}}, \zeta^{l-b_{1}}, \cdots, \zeta^{b_{m}}, \zeta^{l-b_{m}}, \overbrace{1, \ldots, 1}^{r}, \overbrace{-1, \ldots,-1}^{s} \quad\left(0<b_{i}<l\right) .
$$

Then $\mathbf{a}_{\tau}(\rho)=2 m+s$, while

$$
\mathbf{v}_{\tau}(\rho)=\frac{1}{l} \sum_{i=1}^{m}\left(b_{i}+\left(l-b_{i}\right)\right)+\frac{1}{l} \sum_{j=1}^{s} \frac{l}{2}=m+\frac{s}{2} .
$$

We have proved that $\mathbf{v}_{\tau}=\mathbf{a}_{\tau} / 2$.

Since nontrivial eigenvalues of $g \in \mathrm{GL}_{n}\left(\mathcal{O}_{K}\right) \subset \mathrm{GL}_{n}(K)$ stay nontrivial by passing to the residue field $\kappa$, we have

$$
\overline{\mathbf{t}}_{\tau}(\rho)=\operatorname{codim}\left(\kappa^{n}\right)^{g}=\operatorname{codim}\left(K^{n}\right)^{g}=\mathbf{t}_{\tau}(\rho) .
$$

This proves the remaining equalities.

Corollary 4.6. Provided that $\sharp \Gamma$ is prime to the residue characteristic, we have that for any $\tau$,

$$
\mathbf{w}_{\tau \oplus \tau^{\vee}}=\mathbf{a}_{\tau} .
$$

Proof. Since $\tau \oplus \tau^{\vee}$ is balanced,

$$
\mathbf{w}_{\tau \oplus \tau^{\vee}}=\frac{1}{2} \cdot \mathbf{a}_{\tau \oplus \tau^{\vee}} .
$$

Since $\mathbf{a}_{\bullet}$ is additive and $\mathbf{a}_{\tau}=\mathbf{a}_{\tau^{\vee}}$, the corollary follows.

Remark 4.7. Without the tameness condition, $\mathbf{t}_{\bullet}$ and $\overline{\mathbf{t}}_{\bullet}$ do not generally coincide. For instance, if $K=\kappa((t))$ with $\kappa$ a perfect field of characteristic $p>0$, then for the representation

$$
\tau: \mathbb{Z} / p \stackrel{\sim}{\rightarrow}\left\langle\left(\begin{array}{ll}
1 & t \\
0 & 1
\end{array}\right)\right\rangle \subset \mathrm{GL}_{2}(\kappa[[t]])
$$

and $\rho \in S_{K, \mathbb{Z} / p}$ defining a ramified $\mathbb{Z} / p$-extension of $K$, we have

$$
\mathbf{t}_{\tau}(\rho)=1 \text { and } \overline{\mathbf{t}}_{\tau}(\rho)=0 .
$$


4.2. Permutation representations. We now drop the requirement that $\sharp \Gamma$ is prime to the residue characteristic of $K$, and suppose instead that $\tau: \Gamma \rightarrow \mathrm{GL}_{n}\left(\mathcal{O}_{K}\right)$ is a permutation representation. If it is tame, then it is balanced and Proposition 4.5 applies. Otherwise, the assertion of the proposition fails. However some equalities are still valid.

Theorem 4.8. For a permutation representation $\tau$, we have

$$
\overline{\mathbf{t}}_{\tau}=\mathbf{t}_{\tau} \text { and } \mathbf{v}_{\tau}=\frac{1}{2} \cdot \mathbf{a}_{\tau} \text {. }
$$

Proof. The first equality is obvious. For the second, let $\rho \in S_{K, \Gamma}$. In order to compute both sides of the second equality, since $\mathbf{a}$. and $\mathbf{v}$. are complete, we may reduce to the case where $\tau$ is an inclusion map, $\Gamma$ transitively acts on $\{1, \ldots, n\}$ and $\rho$ is surjective. Let $T_{\rho}=\operatorname{Spec} M_{\rho} \rightarrow \operatorname{Spec} K$ be the $\Gamma$-torsor corresponding to $\rho$. From the $\Gamma$-action on $\{1, \ldots, n\}, \rho$ defines also an étale extension $E_{\rho} / K$ of degree $n$. From the assumption we have just made, $M_{\rho}$ and $E_{\rho}$ are fields. Let $\Delta \subset \Gamma$ be the stabilizer of $1 \in\{1, \ldots, n\}$. Then we can identify $E_{\rho}$ with the $\Delta$-invariant subfield $M_{\rho}^{\Delta} \subset M_{\rho}$. Let $\sigma_{i} \in \Delta, i=1, \ldots, n$, be such that $\sigma_{i}(1)=i$. Then, by definition,

$$
\Xi_{\rho}=\left\{\left(\sigma_{1}(e), \ldots, \sigma_{n}(e)\right) \in \mathcal{O}_{M_{\rho}}^{\oplus n} \mid e \in \mathcal{O}_{E_{\rho}}\right\} .
$$

Note that $\sigma_{i}(e)$ is independent of the choice of $\sigma_{i}$. If $e_{1}, \ldots, e_{n}$ are a basis of $\mathcal{O}_{E_{\rho}}$ as an $\mathcal{O}_{K}$-module, then $\left(\sigma_{1}\left(e_{i}\right), \ldots, \sigma_{n}\left(e_{i}\right)\right), i=1, \ldots, n$, are a basis of $\Xi_{\rho}$. Since

$$
d_{E_{\rho} / K}=\operatorname{det}\left(\sigma_{j}\left(e_{i}\right)\right)^{2},
$$

we have

$$
\mathbf{v}_{\tau}(\rho)=\frac{1}{n !} \cdot v_{M_{\rho}}\left(\operatorname{det}\left(\sigma_{j}\left(e_{i}\right)\right)\right)=\frac{1}{2} \cdot v_{K}\left(d_{E_{\rho} / K}\right) .
$$

Lemma 2.6 completes the proof.

Corollary 4.9. For a permutation representation $\tau$, let $2 \tau:=\tau \oplus \tau$. Then

$$
\mathbf{w}_{2 \tau}=2 \cdot \mathbf{t}_{\tau}-\mathbf{a}_{\tau}=\mathbf{t}_{\tau}-\mathbf{s}_{\tau} .
$$

For permutation representations, the expression $\mathbf{w}_{2 \tau}=\mathbf{t}_{\tau}-\mathbf{s}_{\tau}$ shows that the tame part contributes positively to the weight, while the $p$-part contributes negatively, as observed in Yas in some other cases. It is in contrast with that both the tame and $p$ - parts contribute positively to the Artin conductor: $\mathbf{a}_{\tau}=\mathbf{t}_{\tau}+\mathbf{s}_{\tau}$.

Example 4.10. Theorem 4.8 and Corollary 4.9 fail without the assumption that $\tau$ is a permutation representation. Suppose that $K$ has characteristic $p>0$ and $\Gamma=\langle\gamma\rangle \cong \mathbb{Z} /(p)$. For $n \leq p$, consider the representation $\tau: \Gamma \rightarrow \mathrm{GL}_{n}\left(\mathcal{O}_{K}\right)$ with

$$
\tau(\gamma)=\left(\begin{array}{ccccc}
1 & 1 & & & \\
& 1 & 1 & & \\
& & \ddots & \ddots & \\
& & & 1 & 1 \\
& & & & 1
\end{array}\right) .
$$

For $\rho \in S_{K, \Gamma}$ with $L_{\rho} / K$ ramified, let $j$ be the largest integer $i$ with $G_{i} \neq 1$. By an easy calculation, we have

$$
\begin{gathered}
\mathbf{a}_{\tau}(\rho)=(j+1)(n-1) \text { and } \\
\mathbf{t}_{\tau}(\rho)=\overline{\mathbf{t}}_{\tau}(\rho)=n-1 .
\end{gathered}
$$


On the other hand, from [Yas, Example 6.8], we have

$$
\mathbf{w}_{\tau}(\rho)=-\sum_{a=1}^{n-1}\left\lfloor\frac{j a}{p}\right\rfloor .
$$

For instance, if $n=2$, then

$$
2 \mathbf{t}_{\tau}-\mathbf{a}_{\tau}=1-j
$$

while

$$
\mathbf{w}_{2 \tau}=-2\left\lfloor\frac{j}{p}\right\rfloor .
$$

They are not equal unless $p=2$.

\section{The McKay CORRespondence}

In this section, we discuss several versions of the McKay correspondence in terms of motivic invariants. We also formulate point counting realizations of these correspondences and prove them in some cases.

5.1. The Grothendieck ring of varieties and the point counting realization. We first describe the set to which motivic invariants belong. Let $\kappa$ be a perfect field. The Grothendieck ring of $\kappa$-varieties, denoted $K_{0}\left(\operatorname{Var}_{\kappa}\right)$, is the free abelian group of isomorphic classes $[V]$ of $\kappa$-varieties modulo the following relation: if $W$ is a closed subvariety of $V$, then $[V]=[W]+[V \backslash W]$. This indeed becomes a ring by the multiplication defined by $[V] \cdot\left[V^{\prime}\right]:=\left[V \times_{\kappa} V^{\prime}\right]$. For a constructible subset $C \subset V$, its class $[C]$ in $K_{0}\left(\operatorname{Var}_{\kappa}\right)$ is defined in a natural way. We define a distinguished element $\mathbb{L}:=\left[\mathbb{A}_{\kappa}^{1}\right]$ of $K_{0}\left(\operatorname{Var}_{\kappa}\right)$. If $\kappa=\mathbb{F}_{q}$, then there exists a unique ring map

$$
\sharp: K_{0}\left(\operatorname{Var}_{\kappa}\right) \rightarrow \mathbb{Z}
$$

sending $[V]$ to $\sharp V(\kappa)$, which is called the point counting realization.

Motivic integrals which we will consider below take values in a certain modification of $K_{0}\left(\operatorname{Var}_{\kappa}\right)$, the semiring $\mathfrak{R}^{1 / r}$ in [Yas06, Section 3.8], where $r$ is the order of the given finite group $\Gamma$. Alternatively, we may use the complete Grothendieck ring of mixed $G_{\kappa}$-representations over $\mathbb{Q}_{l}, \hat{K}_{0}\left(M R\left(G_{\kappa}, \mathbb{Q}_{l}\right)\right)$, with $l$ a prime number prime to $r$ and to the characteristic of $\kappa$. As in $K_{0}\left(\operatorname{Var}_{\kappa}\right)$, there exists an element $[V] \in \mathfrak{R}^{1 / r}$ associated to each $\kappa$-variety $V$. If $W$ is a closed subvariety of $V$, then we again have $[V]=[W]+[V \backslash W]$. The product of elements $[V]$ and $\left[V^{\prime}\right]$ is similarly given. Moreover $\mathfrak{R}^{1 / r}$ contains fractional powers $\mathbb{L}^{i}$ of $\mathbb{L}=\left[\mathbb{A}_{\kappa}^{1}\right]$ for $i \in \frac{1}{r} \mathbb{Z}$. For $i \in \frac{1}{r} \mathbb{Z}$ with $i>0$, the infinite sum

$$
1+\mathbb{L}^{-i}+\mathbb{L}^{-2 i}+\cdots
$$

converges in $\mathfrak{R}^{1 / r}$. Let $\mathfrak{R}_{0}^{1 / r}$ be the subsemiring of $\mathfrak{R}^{1 / r}$ generated by the classes $[V]$ of $\kappa$-varieties, fractional powers $\mathbb{L}^{i}, i \in \frac{1}{r} \mathbb{Z}$ of $\mathbb{L}$ and the infinite sums of the above form. We then have the point counting realization,

$$
\sharp: \mathfrak{R}_{0}^{1 / r} \rightarrow \mathbb{R},
$$

which sends $[V]$ to $\sharp V(\kappa), \mathbb{L}^{-i}$ to $q^{-i}$, and the infinite sum $1+\mathbb{L}^{-i}+\mathbb{L}^{-2 i}+\cdots$ to

$$
1+q^{-i}+q^{-2 i}+\cdots=\frac{1}{1-q^{-i}} .
$$


We can check that this map is actually well defined for instance by using the realization map $\mathfrak{R}^{1 / r} \rightarrow \hat{K}_{0}\left(M R\left(G_{\kappa}, \mathbb{Q}_{l}\right)\right.$ ) given in [Yas06, Section 3.8] (see also Yas14, page 1142]).

5.2. The tame case. The classical McKay correspondence is about ADE surface singularities over $\mathbb{C}$ and originates in McKay's observation in the late 70 that the same Dynkin diagram appears both in the minimal resolution of singularities and in representation theory. Later, it has been refined and generalized in diverse directions. The first hint of a generalization to higher dimensions came from physics DHVW86, DHVW85. For more details on the McKay correspondence in general, we refer the reader to a nice survey paper Rei02.

A version of the McKay correspondence in terms of motivic invariants was obtained by Batyrev Bat99, where the base field was $\mathbb{C}$. His result was a considerable refinement of the "Physicists' Euler number conjecture". A slightly different version was obtained by Denef-Loeser DL02 over a field of characteristic zero containing all $\sharp \Gamma$-th roots of unity with $\Gamma$ the given finite group. Yasuda Yas06 then generalized it to an arbitrary perfect field of characteristic prime to $\sharp \Gamma$. Now we recall these results. For the sake of intelligibility, we first see the case of a perfect field (possibly of positive characteristic) containing all $\sharp \Gamma$-th roots of unity.

Let $\kappa$ be a perfect field and $\tau: \Gamma \hookrightarrow \mathrm{GL}_{n}(\kappa)$ a faithful representation of a finite group $\Gamma$, through which we regard $\Gamma$ as a subgroup of $\mathrm{GL}_{n}(\kappa)$. The group $\Gamma$ acts on the polynomial ring $\kappa\left[x_{1}, \ldots, x_{n}\right]$ by naturally extending the $\Gamma$-action on the linear part $\bigoplus_{i} \kappa \cdot x_{i} \cong \kappa^{n}$. Let $\mathbb{A}_{\kappa}^{n}:=\operatorname{Spec} \kappa\left[x_{1}, \ldots, x_{n}\right]$ and $X:=\mathbb{A}_{\kappa}^{n} / \Gamma$ the quotient scheme. Since $X$ is a $\mathbb{Q}$-Gorenstein variety over $\kappa$, for a resolution of singularities $f: Y \rightarrow X$, we can define the relative canonical divisor $K_{Y / K}:=K_{Y}-f^{*} K_{X}$, which is a $\mathbb{Q}$-divisor with support contained in the exceptional locus. We say that $f$ is crepant if $K_{Y / K}=0$. We let $0 \in X(\kappa)$ be the image of the origin of $\mathbb{A}_{\kappa}^{n}$.

We suppose that $\kappa$ contains all $\sharp \Gamma$-th roots of unity and choose a primitive $\sharp \Gamma$-th $\operatorname{root} \zeta$.

Definition 5.1. For $g \in \Gamma \subset \mathrm{GL}_{n}(\kappa)$, if it is equivalent to the diagonal matrix $\operatorname{diag}\left(\zeta^{b_{1}}, \ldots, \zeta^{b_{n}}\right)$ with $1 \leq b_{i} \leq \sharp \Gamma$, we put

$$
\mathbf{w}_{\tau}(g):=n-\frac{1}{\sharp \Gamma} \sum_{i=1}^{n} b_{i} \in \mathbb{Q} .
$$

The rational number $\mathbf{w}_{\tau}(g)$ depends only on $\tau$ and the conjugacy class of $g$. We let $\operatorname{Conj}(\Gamma)$ be the set of conjugacy classes of $\Gamma$. The following is the McKay correspondence in the simplest case:

Theorem 5.2 ([DL2, Yas06]). Suppose that $\Gamma \subset \mathrm{GL}_{n}(\kappa)$ has no pseudo-reflection. Namely, for any $g \in \Gamma \backslash\{1\}$, the fixed point locus $\left(\kappa^{n}\right)^{g}$ has codimension at least two. Suppose that $\sharp \Gamma$ is prime to the characteristic of $\kappa$ and that $\kappa$ contains all $\sharp \Gamma$-th roots of unity. Then, for a crepant resolution $f: Y \rightarrow X$, we have

$$
\left[f^{-1}(0)\right]=\sum_{[g] \in \operatorname{Conj}(\Gamma)} \mathbb{L}^{\mathbf{w}_{\tau}(g)} \in \mathfrak{R}_{0}^{1 / \sharp \Gamma} .
$$

When $\kappa$ has characteristic zero and $\Gamma$ is contained in $\mathrm{SL}_{n}(\kappa)$, the theorem was proved in DL02. To deduce the general case of the theorem from a result in Yas06, we need to first prove Theorem 5.9 below. 
Remark 5.3. The weight $\mathbf{w}_{\tau}(g)$ defined above in the tame case is essentially the same thing as what is called age [R96] or fermion number shift [Zas93].

To generalize the result to the tame case over an arbitrary perfect base field, we need the notion of twisted 0-jets from the paper [Yas06], and in particular, need to use Deligne-Mumford stacks.

Let $l$ be a positive integer prime to the characteristic of $\kappa$. For a DeligneMumford stack $\mathcal{Z}$ over $\kappa$, the stack of twisted 0 -jets of order $l, \mathcal{J}_{0}^{l} \mathcal{Z}$, is the DeligneMumford stack whose points are given as follows. Let $\mu_{l, \kappa}:=\operatorname{Spec} \kappa[t] /\left(t^{l}-1\right)$ be the group scheme of $l$-th roots of unity defined over $\kappa$. For a $\kappa$-algebra $R$, an $R$-point of $\mathcal{J}_{0}^{l} \mathcal{Z}$ is a representable $\kappa$-morphism of the form

$$
\left[\operatorname{Spec} R / \mu_{l, \kappa}\right] \rightarrow \mathcal{Z},
$$

where $\mu_{l, \kappa}$ acts on $\operatorname{Spec} R$ trivially and $\left[\operatorname{Spec} R / \mu_{l, \kappa}\right.$ ] is the associated quotient stack. The natural morphism $\operatorname{Spec} R \rightarrow\left[\operatorname{Spec} R / \mu_{l, \kappa}\right]$ gives a natural morphism $\mathcal{J}_{0}^{l} \mathcal{Z} \rightarrow \mathcal{Z}$.

Remark 5.4. Twisted 0 -jets are a special case of twisted $n$-jets given by representable morphisms

$$
\left[\left(\operatorname{Spec} R[t] /\left(t^{n l+1}\right)\right) / \mu_{l, \kappa}\right] \rightarrow \mathcal{Z} .
$$

Twisted jets and twisted arcs, which are representable morphisms

$$
\left[\operatorname{Spec} R[[t]] / \mu_{l, \kappa}\right] \rightarrow \mathcal{Z},
$$

naturally appeared in a generalization of motivic integration to Deligne-Mumford stacks.

Let $\mathcal{X}:=\left[\mathbb{A}_{\kappa}^{n} / \Gamma\right]$ be the quotient stack associated to the $\Gamma$-action on $\mathbb{A}_{\kappa}^{n}$. The natural morphism $\mathbb{A}_{\kappa}^{n} \rightarrow X$ factors through $\mathcal{X}$ and the induced morphism $\mathcal{X} \rightarrow X$ is finite and birational. We put

$$
\mathcal{J}_{0} \mathcal{X}:=\bigsqcup_{p \nmid l} \mathcal{J}_{0}^{l} \mathcal{X}
$$

Since $\mathcal{J}_{0}^{l} \mathcal{X}$ is empty for $l \nmid \sharp \Gamma$, the disjoint union is a finite union.

Remark 5.5. The stack $\mathcal{J}_{0} \mathcal{X}$ is a twisted form of the inertia stack $I \mathcal{X}:=\mathcal{X} \times \Delta, \mathcal{X} \times \mathcal{X}, \Delta$ $\mathcal{X}$ with $\Delta: \mathcal{X} \rightarrow \mathcal{X} \times \mathcal{X}$ the diagonal morphism. Namely $\mathcal{J}_{0} \mathcal{X}$ and $I \mathcal{X}$ become isomorphic after the base change from $\kappa$ to an algebra closure $\bar{\kappa}$.

We put $\left(\mathcal{J}_{0} \mathcal{X}\right)_{0}$ to be the fiber product $\left(\mathcal{J}_{0} \mathcal{X}\right) \times_{X}$ Spec $\kappa$ with respect to the composition morphism $\mathcal{J}_{0} \mathcal{X} \rightarrow \mathcal{X} \rightarrow X$ and the point $0 \in X(\kappa)$. The stack $\left(\mathcal{J}_{0} \mathcal{X}\right)_{0}$ is a closed substack of $\mathcal{J}_{0} \mathcal{X}$. We denote its coarse moduli space by $\overline{\left(\mathcal{J}_{0} \mathcal{X}\right)_{0}}$, which is a scheme finite over $\kappa$. For an algebraic closure $\bar{\kappa}$ of $\kappa$, we have an identification

$$
\overline{\left(\mathcal{J}_{0} \mathcal{X}\right)_{0}}(\bar{\kappa})=\left(\mathcal{J}_{0} \mathcal{X}\right)_{0}(\bar{\kappa}) / \cong \text {. }
$$

Here the right hand side is the set of isomorphism classes of morphisms $\operatorname{Spec} \bar{\kappa} \rightarrow$ $\left(\mathcal{J}_{0} \mathcal{X}\right)_{0}$.

Identifying Spec $\bar{\kappa}$ with the origin of $\mathbb{A}_{\bar{\kappa}}^{n}$, we can identify $[\operatorname{Spec} \bar{\kappa} / \Gamma]$ with a closed substack of $\mathcal{X} \otimes_{\kappa} \bar{\kappa}$. Then $\overline{\left(\mathcal{J}_{0} \mathcal{X}\right)_{0}}(\bar{\kappa})$ is identified with the isomorphism classes of representable morphisms over $\kappa$

$$
\left[\operatorname{Spec} \bar{\kappa} / \mu_{l}\right] \rightarrow[\operatorname{Spec} \bar{\kappa} / \Gamma]
$$


for $l \mid \sharp \Gamma$. Here $\mu_{l}$ is the group of $l$ th roots of unity in $\bar{\kappa}$ and later identified with the group scheme $\mu_{l, \bar{\kappa}}$. Since the automorphism groups of the $\bar{\kappa}$-points of these quotient stacks are $\mu_{l}$ and $\Gamma$ respectively, the morphism gives a homomorphism

$$
\mu_{l} \rightarrow \Gamma,
$$

which is injective because the stack morphism is representable. Now two representable morphisms $\left[\operatorname{Spec} \bar{\kappa} / \mu_{l}\right] \rightrightarrows[\operatorname{Spec} \bar{\kappa} / \Gamma]$ are isomorphic if and only if the corresponding maps $\mu_{l} \rightrightarrows \Gamma$ are conjugate to each other in $\Gamma$. Let $\operatorname{Conj}\left(\mu_{l}, \Gamma\right)$ be the set of injective homomorphisms modulo conjugation in $\Gamma$. We thus have a natural one-to-one correspondence

$$
\overline{\left(\mathcal{J}_{0} \mathcal{X}\right)_{0}}(\bar{\kappa}) \leftrightarrow \bigsqcup_{l \mid \sharp \Gamma} \operatorname{Conj}\left(\mu_{l}, \Gamma\right) .
$$

We fix a primitive $\sharp \Gamma$-th root $\zeta \in \bar{\kappa}$ so that we obtain canonical choices of generators of $\mu_{l}$ for all $l \mid \sharp \Gamma$, that is, $\zeta_{l}:=\zeta^{\sharp \Gamma / l}$. Then we can identify an injective map $\mu_{l} \rightarrow \Gamma$ with the image of $\zeta_{l}$ in $\Gamma$, and $\bigsqcup_{l \mid \sharp \Gamma} \operatorname{Conj}\left(\mu_{l}, \Gamma\right)$ with $\operatorname{Conj}(\Gamma)$. Thus we obtain a one-to-one correspondence

$$
\overline{\left(\mathcal{J}_{0} \mathcal{X}\right)_{0}}(\bar{\kappa}) \leftrightarrow \operatorname{Conj}(\Gamma) .
$$

For later use, we would like to know what subset of $\operatorname{Conj}(\Gamma)$ corresponds to the subset of $\kappa$-points, $\overline{\left(\mathcal{J}_{0} \mathcal{X}\right)_{0}}(\kappa) \subset \overline{\left(\mathcal{J}_{0} \mathcal{X}\right)_{0}}(\bar{\kappa})$, when $\kappa$ is finite. If $q$ is the cardinality of a finite field $\kappa$, then we put

$$
\mathcal{C}_{\Gamma, q}:=\left\{[g] \in \operatorname{Conj}(\Gamma) \mid[g]=\left[g^{q}\right]\right\} \subset \operatorname{Conj}(\Gamma) .
$$

Lemma 5.6. Suppose $\kappa=\mathbb{F}_{q}$. By the correspondence $\overline{\left(\mathcal{J}_{0} \mathcal{X}\right)_{0}}(\bar{\kappa}) \leftrightarrow \operatorname{Conj}(\Gamma)$, the subset $\overline{\left(\mathcal{J}_{0} \mathcal{X}\right)_{0}}(\kappa)$ corresponds to $\mathcal{C}_{\Gamma, q}$.

Proof. For a scheme $U$ over $\mathbb{F}_{q}$, we denote by $F_{U}$ the Frobenius morphism $U \rightarrow U$ defined by the sheaf morphism $\mathcal{O}_{U} \rightarrow \mathcal{O}_{U}, f \mapsto f^{q}$. We define a self-map $F$ of $\overline{\left(\mathcal{J}_{0} \mathcal{X}\right)_{0}}(\bar{\kappa})$ by

$$
F(x):=x \circ F_{\mathrm{Spec} \bar{\kappa}}=F_{\overline{\left(\mathcal{J}_{0} \mathcal{X}\right)_{0}}} \circ x .
$$

The set of $\kappa$-points, $\overline{\left(\mathcal{J}_{0} \mathcal{X}\right)_{0}}(\kappa)$, is the fixed point locus $\overline{\left(\mathcal{J}_{0} \mathcal{X}\right)_{0}}(\bar{\kappa})^{F}$ of $F$. It suffices to show that the self-map $F$ of $\overline{\left(\mathcal{J}_{0} \mathcal{X}\right)_{0}}(\bar{\kappa})$ corresponds to the self-map of $\mathcal{C}_{\Gamma, q}$ given by $[g] \mapsto\left[g^{q}\right]$. Note that Deligne-Mumford stacks over $\kappa$ also have Frobenius morphisms, since for any étale morphism $V \rightarrow U$ of $\kappa$-schemes, $F_{V}$ is the base change of $F_{U}$. Let $x \in \overline{\left(\mathcal{J}_{0} \mathcal{X}\right)_{0}}(\bar{\kappa})$ be a point corresponding to a morphism

$$
\alpha:\left[\operatorname{Spec} \bar{\kappa} / \mu_{l}\right] \rightarrow[\operatorname{Spec} \bar{\kappa} / \Gamma]
$$

and to a homomorphism

$$
\beta: \mu_{l} \rightarrow \Gamma \text {. }
$$

Then $F(x)$ corresponds to the composition of $\alpha$ and the Frobenius morphism of $\left[\operatorname{Spec} \bar{\kappa} / \mu_{l}\right]$, and to the composite map of groups

$$
\mu_{l} \stackrel{F_{\mu_{l}}}{\rightarrow} \mu_{l} \stackrel{\beta}{\rightarrow} \Gamma
$$

where $F_{\mu_{l}}$ is the Frobenius morphism of the group scheme $\mu_{l}=\mu_{l, \bar{\kappa}}$. Since $F_{\mu_{l}}$ sends $\xi \in \mu_{l}$ to $\xi^{q}$, the lemma follows. 
Corollary 5.7. If $\kappa$ contains all $\sharp \Gamma$-th roots of unity, then $\overline{\left(\mathcal{J}_{0} \mathcal{X}\right)_{0}}(\kappa)=\overline{\left(\mathcal{J}_{0} \mathcal{X}\right)_{0}}(\bar{\kappa})$ and we have a one-to-one correspondence

$$
\left\{\text { connected components of } \overline{\left(\mathcal{J}_{0} \mathcal{X}\right)_{0}}\right\} \leftrightarrow \overline{\left(\mathcal{J}_{0} \mathcal{X}\right)_{0}}(\kappa) \leftrightarrow \operatorname{Conj}(\Gamma) .
$$

Proof. Let $q$ be the cardinality of $\kappa$. The condition that $\kappa$ contains all $\sharp \Gamma$-th roots of unity implies that $\sharp \Gamma$ divides $q-1$. Therefore, for any $g \in \Gamma$, we have $g^{q-1}=1$. It follows that the self-map $[g] \mapsto\left[g^{q}\right]$ of $\operatorname{Conj}(\Gamma)$ is the identity map. This shows the correspondence $\overline{\left(\mathcal{J}_{0} \mathcal{X}\right)_{0}}(\kappa) \leftrightarrow \operatorname{Conj}(\Gamma)$ and the equality $\overline{\left(\mathcal{J}_{0} \mathcal{X}\right)_{0}}(\kappa)=\overline{\left(\mathcal{J}_{0} \mathcal{X}\right)_{0}}(\bar{\kappa})$. Hence the associated reduced scheme of $\overline{\left(\mathcal{J}_{0} \mathcal{X}\right)_{0}}(\kappa)$ is the union of $\sharp \operatorname{Conj}(\Gamma)$ copies of Spec $\kappa$, which proves the other correspondence.

We define a function $\mathbf{w}_{\tau}$ on $\overline{\left(\mathcal{J}_{0} \mathcal{X}\right)_{0}}(\bar{\kappa})$ as the function corresponding to $\mathbf{w}_{\tau}$ on $\operatorname{Conj}(\Gamma)$ through the correspondence above.

Remark 5.8. The resulting map $\mathbf{w}_{\tau}: \overline{\left(\mathcal{J}_{0} \mathcal{X}\right)_{0}}(\bar{\kappa}) \rightarrow \mathbb{Q}$ is the same as a restriction of the function sht : $\left|\mathcal{J}_{0} \mathcal{X}\right| \rightarrow \mathbb{Q}$ defined in [Yas06, Section 3.9] up to an involution of $\overline{\left(\mathcal{J}_{0} \mathcal{X}\right)_{0}}(\bar{\kappa})$. The involution corresponds the involution $[g] \leftrightarrow\left[g^{-1}\right]$ of $\operatorname{Conj}(\Gamma)$, and originates in the fact that tangent spaces are considered in the cited paper, while we are implicitly considering cotangent spaces. However the involution does not affect the counting problem, and can be safely ignored.

The value $\mathbf{w}_{\tau}$ of this function actually depends only on the connected component of $\overline{\left(\mathcal{J}_{0} \mathcal{X}\right)_{0}}$ to which the given point belongs. Therefore we write it as $\mathbf{w}_{\tau}(C)$ for the connected component $C$.

Theorem 5.9 (Yas06]). Let $\kappa$ be an arbitrary perfect field. Suppose that $\sharp \Gamma$ is prime to the characteristic of $\kappa$ and that $\Gamma \subset \mathrm{GL}_{n}(\kappa)$ has no pseudo-reflection. For a crepant resolution $f: Y \rightarrow X$, we have

$$
\left[f^{-1}(0)\right]=\sum_{C \subset \overline{\left(\mathcal{J}_{0} \mathcal{X}\right)_{0}}}[C] \cdot \mathbb{L}^{\mathbf{w}_{\tau}(C)} \in \mathfrak{R}_{0}^{1 / \sharp \Gamma} .
$$

Proof. For a positive integer $r$ with $r K_{X}$ Cartier (for instance, $r=\sharp \Gamma$ ), we define an ideal sheaf $\mathcal{G} \subset \mathcal{O}_{X}$ by

$$
\operatorname{Im}\left(\left(\Omega_{X}^{d}\right)^{\otimes r} \rightarrow \mathcal{O}_{X}\left(r K_{X}\right)\right)=\mathcal{G} \cdot \mathcal{O}_{X}\left(r K_{X}\right) .
$$

Let $J_{\infty} X$ be the arc space of $X$, parameterizing morphisms Spec $\kappa[[t]] \rightarrow X$, and $\pi_{0}: J_{\infty} X \rightarrow X$ the projection. We define an invariant by a motivic integral as follows:

$$
M:=\int_{\pi_{0}^{-1}(0)} \mathbb{L}^{\frac{1}{r} \operatorname{ord} \mathcal{G}} d \mu_{X} .
$$

Let $h$ be either $f$ or the morphism $\mathcal{X} \rightarrow X$. Since $h$ is a crepant resolution (in the category of Deligne-Mumford stacks), a standard argument (see the proof of [Yas06, Proposition 90]) shows that

$$
\left(\frac{1}{r} \operatorname{ord} \mathcal{G}\right) \circ h_{\infty}=\operatorname{ord} \mathrm{Jac}_{h},
$$

where $h_{\infty}$ is the map of (twisted) arc spaces associated to $h$ and $\mathrm{Jac}_{h}$ is the Jacobian ideal sheaf of $h$ on $Y$ or $\mathcal{X}$. Applying the transformation rule [Yas06, Theorem 66] 
to $f$, we get

$$
\begin{aligned}
& M=\int \pi_{0}^{-1}\left(f^{-1}(0)\right) \mathbb{L}^{\left(\frac{1}{r} \text { ord } \mathcal{G}\right) \circ f_{\infty}-\operatorname{ord~Jac}_{f}} d \mu_{Y} \\
& =\int \pi_{0}^{-1}\left(f^{-1}(0)\right) d \mu_{Y}=\left[f^{-1}(0)\right] .
\end{aligned}
$$

Let $\pi$ be the projection $\left|\mathcal{J}_{\infty} \mathcal{X}\right| \rightarrow|\mathcal{X}|$ from the space of twisted arcs of $\mathcal{X}$ to the point set of $\mathcal{X}$ and $\mathfrak{s}_{\mathcal{X}}$ is the composition $\left|\mathcal{J}_{\infty} \mathcal{X}\right| \rightarrow\left|\mathcal{J}_{0} \mathcal{X}\right| \stackrel{\text { sht }}{\longrightarrow} \mathbb{Q}$ (see Remark [5.8). Applying the transformation rule to the morphism $\mathcal{X} \rightarrow X$, which we denote by $p$, we get

$$
\begin{aligned}
M & =\int_{\pi^{-1}(0)} \mathbb{L}^{\left(\frac{1}{r} \operatorname{ord} \mathcal{G}\right) \circ p_{\infty}-\operatorname{ord~Jac}_{p}+\mathfrak{s}_{\mathcal{X}}} d \mu_{\mathcal{X}} \\
& =\int_{\pi^{-1}(0)} \mathbb{L}^{\mathfrak{s} \mathcal{X}} d \mu_{\mathcal{X}}=\sum_{C \subset \overline{\left(\mathcal{J}_{0} \mathcal{X}\right)_{0}}}[C] \cdot \mathbb{L}^{\mathbf{w}_{\tau}(C) .}
\end{aligned}
$$

We have proved the theorem.

Remark 5.10. If the fiber product $Y \times_{X} \mathcal{X}$ has a resolution of singularities, then the theorem follows from [Yas06, Corollary 72]. However, since we are working in arbitrary characteristic and do not know the existence of resolution, we avoided to use it.

When $\kappa$ contain all $\sharp \Gamma$-th roots of unity, then $\overline{\left(\mathcal{J}_{0} \mathcal{X}\right)_{0}}$ is the disjoint union of $\sharp \operatorname{Conj}(\Gamma)$ copies of Spec $\kappa$, and we obtain Theorem 5.2 . Applying the point counting realization $\sharp: \mathfrak{R}_{0}^{1 / \sharp \Gamma} \rightarrow \mathbb{R}$, we obtain:

Corollary 5.11. Let $\kappa$ be a finite field. Suppose that $\sharp \Gamma$ is prime to the characteristic of $\kappa$ and that $\Gamma \subset \mathrm{GL}_{n}(\kappa)$ has no pseudo-reflection. For a crepant resolution $f: Y \rightarrow X$, we have

$$
\sharp f^{-1}(0)(\kappa)=\sum_{[g] \in \mathcal{C}_{\Gamma, q}} q^{\mathbf{w}_{\tau}(g)} .
$$

5.3. The wild case. A conjectural generalization of the McKay correspondence to the wild case was formulated by Yasuda Yas, after studying the special case of a cyclic group of prime order Yas14. Actually it was a generalization not only to the wild case but also the relative setting over the integer ring of a local field. Let $K$ be a local field and $\tau: \Gamma \rightarrow \mathrm{GL}_{n}\left(\mathcal{O}_{K}\right)$ a faithful representation of a finite group $\Gamma$. Note that a representation $\Gamma \rightarrow \mathrm{GL}_{n}(\kappa)$ as in the last subsection is considered as a special case by composing it with the inclusion map $\mathrm{GL}_{n}(\kappa) \hookrightarrow \mathrm{GL}_{n}(\kappa[[t]])$. We can similarly construct the quotient scheme $X:=\mathbb{A}_{\mathcal{O}_{K}}^{n} / \Gamma$. We can define the canonical divisor $K_{X}$ over $\mathcal{O}_{K}$ (see [Kol13, page 8]), which is $\mathbb{Q}$-Cartier. For a proper birational morphism $f: Y \rightarrow X$ with $Y$ smooth over $\mathcal{O}_{K}$, we can say that $f$ is a crepant resolution if $K_{Y}=f^{*} K_{X}$.

We need a conjectural moduli space of (not pointed) étale $\Gamma$-torsors of Spec $K$ : we denote it by $\Gamma-\operatorname{Cov}(K)$. The base field of the space should be not $K$ but $\kappa$. In the tame case, $\Gamma-\operatorname{Cov}(K)$ is expected to be zero dimensional. Moreover, if $\kappa$ is algebraically closed, then it should consist of $\sharp \operatorname{Conj}(\Gamma)$ points. On the other hand, in the wild case, the space is expected to be infinite dimensional. A (not pointed) 
$\Gamma$-torsor over Spec $K$ corresponds to a continuous homomorphism $G_{K} \rightarrow \Gamma$ modulo conjugation in $\Gamma$. Through this correspondence, we can define a function

$$
\mathbf{w}_{\tau}: \Gamma-\operatorname{Cov}(K) \rightarrow \mathbb{Q}
$$

corresponding to the function $\mathbf{w}_{\tau}$ on $S_{K, \Gamma}$. We expect that this function has finite dimensional constructible subsets as fibers (at least when $X$ admits a crepant resolution) and expressions $\left[\mathbf{w}_{\tau}^{-1}(r)\right], r \in \mathbb{Q}$ make sense as elements of $\mathfrak{R}^{1 / \sharp \Gamma}$. The motivic integral of $\mathbb{L}^{\mathbf{w}_{\tau}}: \Gamma-\operatorname{Cov}(K) \rightarrow \mathfrak{R}^{1 / r}$ is then defined as

$$
\int_{\Gamma-\operatorname{Cov}(K)} \mathbb{L}^{\mathbf{w}_{\tau}}:=\sum_{r \in \mathbb{Q}}\left[\mathbf{w}_{\tau}^{-1}(r)\right] \mathbb{L}^{r} \in \mathfrak{R}^{1 / \sharp \Gamma} \cup\{\infty\} .
$$

The following conjecture was formulated in Yas, under the assumption that the residue field $\kappa$ is algebraically closed.

Conjecture 5.12. Suppose that for every $g \in \Gamma \backslash\{1\}$, the fixed point locus $\left(\mathbb{A}_{\mathcal{O}_{K}}^{n}\right)^{g}$ has codimension at least two, equivalently that the quotient map $\mathbb{A}_{\mathcal{O}_{K}}^{n} \rightarrow X$ is étale in codimension one. Let $f: Y \rightarrow X$ be a crepant resolution and $Z \subset Y$ be the preimage of the origin $0 \in X(\kappa)$. Then

$$
[Z]=\int_{\Gamma-\operatorname{Cov}(K)} \mathbb{L}^{\mathbf{w}_{\tau}} \in \mathfrak{R}_{0}^{1 / \sharp \Gamma} .
$$

Remark 5.13. One difficulty in formulating the conjecture was to find out the correct function $\mathbf{w}_{\tau}$. In Yas, the function was defined only when $\kappa$ is algebraically closed. Studying a relation with the Artin conductor, we realized that we need to use $T_{\rho^{\mathrm{nr}}}$ rather than $T_{\rho}$ for Definition 3.5

Remark 5.14. In [Yas, the conjecture above was derived as a direct consequence of a conjectural change of variables formula of motivic integrals for the morphism $\left[\mathbb{A}_{\mathcal{O}_{K}}^{n} / \Gamma\right] \rightarrow X$, just as in the tame case. The weight function $\mathbf{w}_{\tau}$ appears in the change of variables formula as necessary shifts coming from twists of twisted arcs. To compute shifts, Yasuda used a technique which he calls untwisting and which reduces a study of twisted arcs to one of ordinary arcs. The tuning submodule $\Xi_{\rho}$ is a key ingredient in this technique. This is why it appears in the definition of the weight.

Remark 5.15. Even if a (crepant) resolution of $X$ does not exist, invariants $\int_{\Gamma-\operatorname{Cov}(K)} \mathbb{L}^{\mathbf{w}_{\tau}}$ and $M\left(K, \Gamma,-\mathbf{w}_{\tau}\right)$ have an important meaning. Indeed they should be equal to stringy invariants of the quotient singularity and contain rich information on the geometry of the singularity.

As mentioned above, if $\kappa$ is algebraically closed and $\sharp \Gamma$ is prime to its characteristic, then $\Gamma-\operatorname{Cov}(K)$ consists of finitely many points, which correspond to conjugacy classes of $\Gamma$. If moreover $K=\kappa((t))$ and $\tau$ is defined over $\kappa$, then we easily see that the conjecture is reduced to Theorem 5.2. When $K=\kappa((t))$ with $\kappa$ a perfect field of characteristic $p>0, \Gamma=\mathbb{Z} / p$ and $\tau$ is defined over $\kappa$, then the conjecture holds with $\Gamma-\operatorname{Cov}(K)$ replaced with a similar parameter space Yas14.

What is the point counting version of the conjecture? The obvious choice for the left side of the equality is $\sharp Z(\kappa)$. The one for the right side is not completely clear. For, there is ambiguity about what kind of space $\Gamma-\operatorname{Cov}(K)$ is: which moduli 
functor it represents, or whether the moduli space is fine or coarse. However, a reasonable choice would be

$$
\sum_{\alpha \in \Gamma-\operatorname{Cov}(K)^{\prime}} \frac{1}{\sharp \operatorname{Aut}(\alpha)} \cdot q^{\mathbf{w}_{\tau}(\alpha)},
$$

where $\Gamma-\operatorname{Cov}(K)^{\prime}$ denotes the set of isomorphism classes of $\Gamma$-torsors over Spec $K$ and $\operatorname{Aut}(\alpha)$ is the automorphism group of a $\Gamma$-torsor $\alpha$. The appearance of the coefficient $1 / \sharp \operatorname{Aut}(\alpha)$ is natural, since it is customary to count objects with weights inverse proportional to the order of the automorphism group.

Lemma 5.16. Sum (5.1) is equal to $M\left(K, \Gamma,-\mathbf{w}_{\tau}\right)$.

Proof. If $H$ is the stabilizer group of a connected component of a $\Gamma$-torsor $\alpha$, then $\operatorname{Aut}(\alpha)$ is the opposite group of the centralizer $C_{\Gamma}(H)$ and there are exactly $\sharp \Gamma / \sharp C_{\Gamma}(H)$ elements of $S_{K, \Gamma}$ corresponding to $\alpha$, which shows the lemma.

We pose the following conjecture as the point counting realization of the last conjecture:

Conjecture 5.17. In addition to the assumption of Conjecture 5.12, we suppose that the residue field $\kappa$ is finite. Then

$$
\sharp Z(\kappa)=M\left(K, \Gamma,-\mathbf{w}_{\tau}\right) .
$$

Again, if $K=\kappa((t))$ with $\kappa$ a perfect field of characteristic $p>0, \Gamma=\mathbb{Z} / p$ and $\tau$ is defined over $\kappa$, then Conjecture 5.17 holds Yas14, Corollary 6.28].

Lemma 5.18. We have

$$
M\left(K, \Gamma,-\mathbf{w}_{\tau}\right)=\sum_{[g] \in \mathcal{C}_{\Gamma, q}} q^{\mathbf{w}_{\tau}(g)} .
$$

Proof. The tame absolute Galois group of $\kappa((t))$ is profinitely generated by two elements, say $a$ and $b$, with exactly one relation: $b a b^{-1}=a^{q}$ [NSW08, page 410]. Therefore there exists a bijection

$$
\begin{aligned}
S_{K, \Gamma} & \rightarrow\left\{(g, h) \in \Gamma^{2} \mid h g h^{-1}=g^{q}\right\} \\
\rho & \mapsto(\rho(a), \rho(b)) .
\end{aligned}
$$

Since $a$ profinitely generates the inertia group, from Lemma 4.3, the map

$$
S_{K, \Gamma} \rightarrow \operatorname{Conj}(\Gamma), \rho \mapsto[\rho(a)]
$$

is compatible with the functions both denoted by $\mathbf{w}_{\tau}$ in Definitions 3.7 and 5.1. It follows that

$$
M\left(K, \Gamma,-\mathbf{w}_{\tau}\right)=\frac{1}{\sharp \Gamma} \cdot \sum_{(g, h) \in \Gamma^{2}: h g h^{-1}=g^{q}} q^{\mathbf{w}_{\tau}(g)} .
$$

For each $g \in \Gamma$, there exist exactly $\sharp C_{\Gamma}(g)$ elements $h \in \Gamma$ such that $h g h^{-1}=g^{q}$. Hence

$$
\begin{aligned}
M\left(K, \Gamma,-\mathbf{w}_{\tau}\right) & =\frac{1}{\sharp \Gamma} \cdot \sum_{g \in \Gamma:[g]=\left[g^{q}\right]} \sharp C_{\Gamma}(g) \cdot q^{\mathbf{w}_{\tau}(g)} \\
& =\sum_{[g] \in \mathcal{C}_{\Gamma, q}} q^{\mathbf{w}_{\tau}(g)} .
\end{aligned}
$$


This lemma and Corollary 5.11 show:

Corollary 5.19. Suppose that $\kappa$ is a perfect field of characteristic prime to $\sharp \Gamma$, that $K=\kappa((t))$, and that $\tau$ factors through $\mathrm{GL}_{n}(\kappa)$. Then Conjecture 5.17 holds.

We note that a closely related result was proved in [Ros07.

Example 5.20. Let $\Gamma=\mathbb{Z} / 3, K$ a local field with a finite residue field $\kappa=\mathbb{F}_{q}$ of characteristic $\neq 3, \tau: \Gamma \rightarrow \mathrm{GL}_{3}\left(\mathcal{O}_{K}\right)$ the regular representation. From Ked07, Example 6.1] and Corollary [4.6, we have

$$
M\left(K, \Gamma,-\mathbf{w}_{\tau}\right)=\left\{\begin{array}{lll}
2 q+1 & (q \equiv 1 & \bmod 3) \\
1 & (q \equiv 2 & \bmod 3) .
\end{array}\right.
$$

Let $X:=\mathbb{A}_{\mathcal{O}_{K}}^{3}$ and $f: Y \rightarrow X$ the blowup along the zero section $\operatorname{Spec} \mathcal{O}_{K} \subset X$. Then $f$ is a crepant resolution. Its pull-back by Spec $\kappa \hookrightarrow \operatorname{Spec} \mathcal{O}_{K}, f_{\kappa}: Y_{\kappa} \rightarrow X_{\kappa}$, depends only on $\kappa$ (independent of $K$ ). Therefore the corollary above shows that

$$
\sharp f^{-1}(0)(\kappa)=M\left(K, \Gamma,-\mathbf{w}_{\tau}\right) .
$$

If $q \equiv 1 \bmod 3$, then indeed $X$ is a normal toric 3-fold and isomorphic to the product of the affine toric surface with $A_{2}$-singularity and the affine line. Hence the exceptional locus of $f_{\kappa}$ is two projective lines meeting at a point, and has $2 q+1$ $\mathbb{F}_{q}$-points. Conversely, if $q \equiv 2 \bmod 3$, then $Z$ has only one $\mathbb{F}_{q}$-point. It shows that $Z$ is irreducible. For, if it was not the case, then an irreducible component would be a Brauer-Severi variety having a rational point and hence isomorphic to the projective line (see $A r t 82$ ), which has more than one $\mathbb{F}_{q}$-point.

5.4. The punctual Hilbert scheme. Though it is hardly known when a crepant resolution exists in the wild case, there is an infinite series of examples. Let $\mathbb{A}_{R}^{2}$ be an affine plane over a ring $R$. The Hilbert scheme of $n$ points of $\mathbb{A}_{R}^{2}$,

$$
H_{R}:=\operatorname{Hilb}^{n}\left(\mathbb{A}_{R}^{2} / \operatorname{Spec} R\right),
$$

is a projective smooth $R$-scheme of dimension $2 n$. Let

$$
X_{R}:=\mathbb{A}_{R}^{2} \times_{R} \cdots \times_{R} \mathbb{A}_{R}^{2} / S_{n}
$$

be the $n$th symmetric product of $\mathbb{A}_{R}^{2}$. We can also think of $X$ as the quotient variety associated to $2 \sigma:=\sigma \oplus \sigma$ with $\sigma$ the defining representation of $S_{n}$ (over $R$ ). Then there is the Hilbert-Chow morphism

$$
H_{R} \rightarrow X_{R}
$$

(for instance, see [Kol96]). Under a reasonable assumption on $R$, in particular, if $R=\mathcal{O}_{K}$ for a local field $K$, then from Bea83, KT01, BK05, this is a crepant resolution. Note that $H_{R}$ and $X_{R}$ are compatible with the base change by any ring homomorphism $R \rightarrow R^{\prime}$.

The following theorem provides further strong evidence for Conjectures 5.12 and 5.17.

Theorem 5.21. Suppose that $K$ is a local field with residue field $\kappa=\mathbb{F}_{q}$ and put $R:=\mathcal{O}_{K}$. Then Conjecture 5.17 holds for the crepant resolution $H_{R} \rightarrow X_{R}$ and the representation $2 \sigma: S_{n} \rightarrow \mathrm{GL}_{2 n}(R)$. Moreover, if $Z$ is the preimage of the origin $0 \in X(\kappa)$, then we have

$$
\sharp Z(\kappa)=M\left(K, \Gamma,-\mathbf{w}_{2 \sigma}\right)=\sum_{m=0}^{n} P(n, n-m) q^{m} .
$$


Proof. From ES87 (see also CV08 for the case of an arbitrary base field), $Z$ is stratified into finitely many affine spaces:

$$
Z=\bigsqcup_{i=1}^{l} \mathbb{A}_{\kappa}^{n_{i}}
$$

Moreover, for each $0 \leq m \leq n-1$, there are exactly $P(n, n-m)$ strata of dimension $m$. In particular, we have

$$
\sharp Z(\kappa)=\sum_{m=0}^{n-1} P(n, n-m) q^{m} .
$$

Let $E=\prod_{i=1}^{l} E_{i}$ be an étale extension of degree $n$ with $E_{i}$ fields and let $e_{i}$ and $f_{i}$ be the ramification and inertia indices of $E_{i} / K$. Then we have the partition of $n$ into exactly $\sum f_{i}$ parts,

$$
n=\overbrace{e_{1}+\cdots+e_{1}}^{f_{1} \text { times }}+\cdots+\overbrace{e_{l}+\cdots+e_{l}}^{f_{l} \text { times }} .
$$

We will denote the partition by $\mathbf{p}(E)$. When $\rho \in S_{K, S_{n}}$ corresponds to $E$, we put $\mathbf{p}(\rho):=\mathbf{p}(E)$. Then Bhargava's formula Bha07, Proposition 2.2] is written as follows, according to Kedlaya's interpretation: for a partition $\mathbf{p}$ of $n$ into exactly $n-m$ parts,

$$
\frac{1}{\sharp \Gamma} \sum_{\substack{\rho \in S_{K, S} \\ \mathbf{p}(\rho)=\mathbf{p}}} q^{-\mathbf{a}_{\sigma}(\rho)}=q^{-m} .
$$

On the other hand, for $\rho$ with $\mathbf{p}(\rho)=\mathbf{p}$,

$$
\mathbf{t}_{\sigma}(\rho)=m \text {. }
$$

Indeed, the induced étale extension $E^{\mathrm{nr}} / K^{\mathrm{nr}}$ is isomorphic to $\prod_{i} L_{i}^{f_{i}}$ with $L_{i} / K$ a

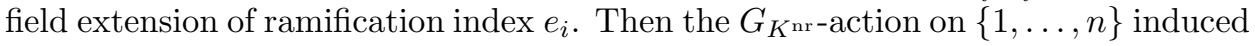
by $\rho$ has $\sum f_{i}$ orbits. Since $\sum f_{i}=n-m$, we have

$$
\mathbf{t}_{\sigma}(\rho)=\operatorname{codim}(\kappa)^{G_{K^{\mathrm{nr}}}}=m .
$$

If we denote by $\sharp \mathbf{p}$ the number of parts of $\mathbf{p}$, then, from Corollary 4.9 , we have

$$
\begin{aligned}
M\left(K, S_{n},-\mathbf{w}_{2 \sigma}\right) & =\frac{1}{n !} \sum_{\mathbf{p}} \sum_{\rho: \mathbf{p}(\rho)=\mathbf{p}} q^{\mathbf{w}_{2 \sigma}(\rho)} \\
& =\frac{1}{n !} \sum_{\mathbf{p}} \sum_{\rho: \mathbf{p}(\rho)=\mathbf{p}} q^{2 \mathbf{t}_{\sigma}(\rho)-\mathbf{a}_{\sigma}(\rho)} \\
& =\sum_{\mathbf{p}} q^{n-\sharp \mathbf{p}} \\
& =\sum_{m=0}^{n-1} P(n, n-m) q^{m} .
\end{aligned}
$$

We have proved the theorem. 


\section{REFERENCES}

[Art82] M. Artin. Brauer-Severi varieties. In Brauer groups in ring theory and algebraic geometry (Wilrijk, 1981), volume 917 of Lecture Notes in Math., pages 194-210. Springer, Berlin, 1982.

[Bat99] Victor V. Batyrev. Non-Archimedean integrals and stringy Euler numbers of logterminal pairs. J. Eur. Math. Soc. (JEMS), 1(1):5-33, 1999.

[Bea83] Arnaud Beauville. Variétés kähleriennes dont la première classe de Chern est nulle. J. Differ. Geom., 18:755-782, 1983.

[Bha07] Manjul Bhargava. Mass formulae for extensions of local fields, and conjectures on the density of number field discriminants. Int. Math. Res. Not. IMRN, (17):Art. ID rnm052, 20, 2007.

[BK05] Michel Brion and Shrawan Kumar. Frobenius splitting methods in geometry and representation theory, volume 231 of Progress in Mathematics. Birkhäuser Boston Inc., Boston, MA, 2005.

[CV08] Aldo Conca and Giuseppe Valla. Canonical Hilbert-Burch matrices for ideals of $k[x, y]$. Michigan Math. J., 57:157-172, 2008. Special volume in honor of Melvin Hochster.

[DHVW85] L. Dixon, J. A. Harvey, C. Vafa, and E. Witten. Strings on orbifolds. Nuclear Phys. $B, 261(4): 678-686,1985$.

[DHVW86] L. Dixon, J. Harvey, C. Vafa, and E. Witten. Strings on orbifolds. II. Nuclear Phys. $B, 274(2): 285-314,1986$.

[DL02] Jan Denef and François Loeser. Motivic integration, quotient singularities and the McKay correspondence. Compositio Math., 131(3):267-290, 2002.

[ES87] Geir Ellingsrud and Stein Arild Strømme. On the homology of the Hilbert scheme of points in the plane. Invent. Math., 87(2):343-352, 1987.

[IR96] Yukari Ito and Miles Reid. The McKay correspondence for finite subgroups of $\mathrm{SL}(3, \mathbf{C})$. In Higher-dimensional complex varieties (Trento, 1994), pages 221-240. de Gruyter, Berlin, 1996.

[Ked07] Kiran S. Kedlaya. Mass formulas for local Galois representations. Int. Math. Res. Not. IMRN, (17):Art. ID rnm021, 26, 2007. With an appendix by Daniel Gulotta.

[Kol96] János Kollár. Rational curves on algebraic varieties, volume 32 of Ergebnisse der Mathematik und ihrer Grenzgebiete. 3. Folge. A Series of Modern Surveys in Mathematics [Results in Mathematics and Related Areas. 3rd Series. A Series of Modern Surveys in Mathematics]. Springer-Verlag, Berlin, 1996.

[Kol13] János Kollár. Singularities of the minimal model program, volume 200 of Cambridge Tracts in Mathematics. Cambridge University Press, Cambridge, 2013. With a collaboration of Sándor Kovács.

[KT01] Shrawan Kumar and Jesper Funch Thomsen. Frobenius splitting of Hilbert schemes of points on surfaces. Math. Ann., 319(4):797-808, 2001.

[Mil80] James S. Milne. Étale cohomology, volume 33 of Princeton Mathematical Series. Princeton University Press, Princeton, N.J., 1980.

[NSW08] Jürgen Neukirch, Alexander Schmidt, and Kay Wingberg. Cohomology of number fields, volume 323 of Grundlehren der Mathematischen Wissenschaften [Fundamental Principles of Mathematical Sciences]. Springer-Verlag, Berlin, second edition, 2008.

[Rei02] Miles Reid. La correspondance de McKay. Astérisque, (276):53-72, 2002. Séminaire Bourbaki, Vol. 1999/2000.

[Ros07] Michael A. Rose. Frobenius action on l-adic Chen-Ruan cohomology. Commun. Number Theory Phys., 1(3):513-537, 2007.

[Ser78] Jean-Pierre Serre. Une "formule de masse" pour les extensions totalement ramifiées de degré donné d'un corps local. C. R. Acad. Sci. Paris Sér. A-B, 286(22):A1031-A1036, 1978.

[Ser79] Jean-Pierre Serre. Local fields, volume 67 of Graduate Texts in Mathematics. SpringerVerlag, New York, 1979. Translated from the French by Marvin Jay Greenberg.

[Ser87] Jean-Pierre Serre. Sur les représentations modulaires de degré 2 de Gal $(\overline{\mathbf{Q}} / \mathbf{Q})$. Duke Math. J., 54(1):179-230, 1987.

[Tag02] Yuichiro Taguchi. Induction formula for the Artin conductors of mod $l$ Galois representations. Proc. Amer. Math. Soc., 130(10):2865-2869 (electronic), 2002. 
[Woo08] Melanie Matchett Wood. Mass formulas for local Galois representations to wreath products and cross products. Algebra Number Theory, 2(4):391-405, 2008.

[WY] Melanie Matchett Wood and Takehiko Yasuda. Mass formulas for local Galois representations and quotient singularities II: dualities and resolution of singularities. in preparation.

[Yas] Takehiko Yasuda. Toward motivic integration over wild Deligne-Mumford stacks. arXiv:1302.2982, to appear in the proceedings of "Higher Dimensional Algebraic Geometry - in honour of Professor Yujiro Kawamata's sixtieth birthday".

[Yas06] Takehiko Yasuda. Motivic integration over Deligne-Mumford stacks. Adv. Math., 207(2):707-761, 2006.

[Yas14] Takehiko Yasuda. The $p$-cyclic McKay correspondence via motivic integration. Compos. Math., 150(7):1125-1168, 2014.

[Zas93] Eric Zaslow. Topological orbifold models and quantum cohomology rings. Comm. Math. Phys., 156(2):301-331, 1993.

Department of Mathematics, University of Wisconsin-Madison, 480 Lincoln Drive, Madison, Wi 53705 USA, and American Institute of Mathematics, 360 Portage Ave, Palo Alto, CA 94306-2244 USA

E-mail address: mmwood@math.wisc.edu

Department of Mathematics, Graduate School of Science, Osaka University, ToyONAKA, OSAKA 560-0043, JAPAN

E-mail address: takehikoyasuda@math.sci.osaka-u.ac.jp 